\title{
COHORT ANALYSIS OF MORTALITY RATES AS AN HISTORICAL OR NARRATIVE TECHNIQUE
}

BY

\author{
R. A. M. CASE \\ The Chester Beatty Research Institute, The Institute of Cancer Research, The Royal Cancer Hospital, London \\ (Reprinted from the British fournal of Preventive E Social Medicine 1956;10:159-71. \\ A commentary by C Osmond follows on page 125)
}

The use of cohort analysis applied to mortality rates in human biology has been limited and obscured by pre-occupation with two concepts which fail to embrace the whole or probably even the larger part of the domain properly described by the method, though each concept is of legitimate interest in its own sphere. These two concepts are:

(1) That the shape of the mortality curve can be adequately described by a mathematical formula expressing a "law of mortality" [e.g. that of Gompertz (1825) or of Makeham (1860)].

(2) That if the mortality pattern either for all mortality or for a particular disease is fixed in some such manner, then the intensity with which the population is affected is determined by the early years of life of the members of that population. This early determination of the mortality pattern is referred to as the "generation" effect.

\section{HISTORY}

If cohort analysis is taken to mean the following of the development of some attribute, in this paper mortality rate, in a particular group of people defined by their birth date, the first unequivocal description of the method that I have been able to trace was that given by Andvord (1930). It related to the mortality from tuberculosis, and dealt with what has been defined as the "generation effect". An earlier paper by Derrick (1927) mentioning the "generation effect" for all mortality does not use cohort analaysis, but a method that will be defined below as "age-contour analysis". A claim made in an editorial footnote in 1953 that Korteweg (1927) had first used the method in a study of mortality rates from tuberculosis is not borne out by reference to Korteweg's paper.

Kermack, McKendrick, and McKinlay $(1934 a, b)$ then take up the tale and emphasize the effect of mortality in the early years of life in determining subsequent mortality. They succeeded in disposing of the gloomy prophecy of Karl Pearson and his school (e.g. Pearson 1912) that decreasing infantile mortality would inevitably increase mortality rates at higher ages because of the lack of proper natural selection.

About the same time Cramér and Wold $(1934,1935)$ had independently been studying the method from an actuarial standpoint, and had published two reports of a detailed cohort analysis of the mortality rates in Sweden. They explicitly referred to the work as a "study in graduating and forecasting". They came to the conclusion, when comparing the results of graduating both the cohort death rates and the "date-contour" death rates by the Makeham formula, the "neither graduation seems to be decidedly to be preferred to the other".

In a valedictory address to the Royal Statistical Society, Greenwood (1936) discussed the method in relation to "English Death Rates, Past, Present and Future", but seemed to be so obsessed by the actuarial demand for graduation and extrapolation that the narrative value of the method escaped him. He stated (p. 691) that ". . . the occurrence of epidemics, such as the cholera epidemics of the past or the influenza of our own time must prejudice arithmetically a diagonal [cohort] method more seriously than a columnar [date-contour] method". (Words in brackets inserted).

Barclay and Kermack (1937-38) extended cohort analysis to birth rates, and this started a development of technique that gave rise to studies, excellently described by Taylor (1952), that have changed our concept of fertility analysis but are not germane to the issues raised in the present paper.

In 1939 came the posthumous publication of work already verbally communicated in 1936 by Frost, where the term "cohort" was used and defined for the first time. This important paper deals with the shape of the mortality curve in tuberculosis and the variations in intensity of mortality rates from this disease according to the date of birth. It was a forerunner of a series of papers on tuberculosis mortality which extended these observations to different countries but were concerned in the main with the "generation effect" (Picken, 1940; Moine and Oudet, 1947; Daw, 1950; Lancaster, 1950; Springett, 1950 and 1952; Spicer, 1954). The most comprehensive and penetrating discussion of cohort analysis that has so far appeared to be found in the fundamental study 
reported by Springett (1950), and this paper should be consulted both for details of the way curves can change in general and the cohort analysis of tuberculosis mortality rates in particular.

Korteweg (1951) used cohort analysis to draw attention to the explanation of the apparently anomalous shape of the age-specific mortality curve for lung cancer, and this was followed by a series of papers (Korteweg, 1953; Levin, 1953; Dorn, 1953; Clemmesen, Nielsen, and Jensen, 1953; Cutler and Loveland, 1954) in which this artefact is referred to, though none of them makes it clear that this is a general effect of the rate of rise exceeding a critical value. Stocks (1953a, b) explored the cancer field more widely by also plotting the mortality curves for cancer of the uterus, female breast, and stomach but was concerned with the hypothesis of Nordling (1953) that cancer is the result of the seventh mutation of a cell, and that this can be deduced from cancer mortality rates. Haenszel and Shimkin (1956) attempted to use the narrative properties of the analytical system to determine whether the social history of smoking habits in the United States of America was compatible with the epidemiology of lung cancer as revealed by cohort analysis.

\section{The Method}

The principle underlying cohort analysis is so simple that an experimental biologist, who would unhesitatingly, if unwittingly, make use of the method in his animal experiments, might be surprised that any demographers feel that discussion of its logical credentials is necessary.

If we take a patch of new-born animals and wish to observe the mortality that occurs when they are kept under specified conditions, we record the number that die during successive periods of time and calculate the mortality rate from this number and from the number of survivors of the original batch. If we were to run a series of experiments, each batch being kept under different conditions, we should not attempt to make a mortality curve by taking mortality rates at different ages from different series of animals.

If our series of experiments is so large that we start each new one at a regular interval, this interval being the same as the intervals between calculating the mortality rates, we may enter the observations on a grid (Fig. 1) in which the top of each column represents the start of an experiment and the successive observations on any one batch of animals lie along a diagonal of the grid. If the grid record includes some experiments that has been started before the arbitrary zero date, all the cells of the grid can be filled with mortality rates. grid:

There are now three ways of traversing this

(1) The diagonal, labelled "cohort", in which each successive square contains a mortality rate derived from the survivors of one experimental batch of animals.
(2) The vertical, labelled "date-contour", in which each successive square contains a mortality rate derived from a different batch of experimental animals, each batch kept under different conditions, although the ages defining each rate form a smooth progression.

(3) The horizontal, labelled "age-contour", in which each square contains a mortality rate derived from a different batch of animals, but in which the age of the animals referred to is the same.

Whilst it would not normally occur to us that the vertical series formed in any sense a biological description of the force of mortality characteristic of the type of animal used, since each rate is from a batch of animals kept by definition under different conditions, we might well use the horizontal series to see if the different experimental conditions had an effect on the mortality rate at any stated age. If, and only if, no effect were found, and the rates along the horizontal line remained constant, the vertical line would become a valid description of the mortality pattern for all the batches of animals, for then the vertical lines and the diagonals would have become identical.

When analogous studies are made on human populations, the form of social accountancy that is perforce used tends to obsecure this issue. In England and Wales, for example, the Registrar-General publishes an "Annual Report", and obviously he can only record the mortality that has occurred in that year, i.e. a series of rates lying along the "date-contour" of Fig. 1. Ideally it would seem that a grid constructed from mortality rates for each year of life, collected annually, would offer the best opportunity for cohort analysis, since the experience of the survivors of those born in each successive calendar year could be followed. In practice, it is not the custom to record mortality regularly at each year of age, but to divide the ages into a series of groups, often of 5 years.

When this is done, a somewhat complex situation occurs because the membership of a group of people who are aged between 50 and 55 in 1950 is not exactly the same as the

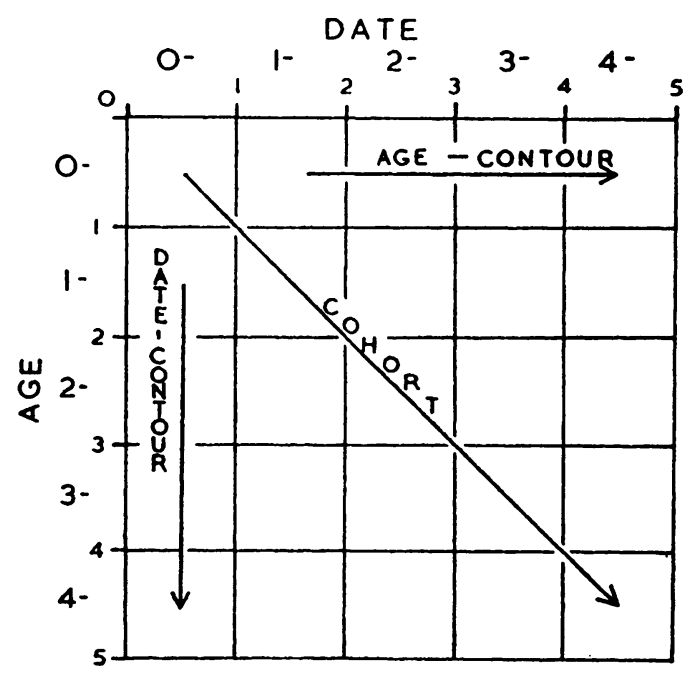

Figure 1 Principle of analysis by cohort and contour. 


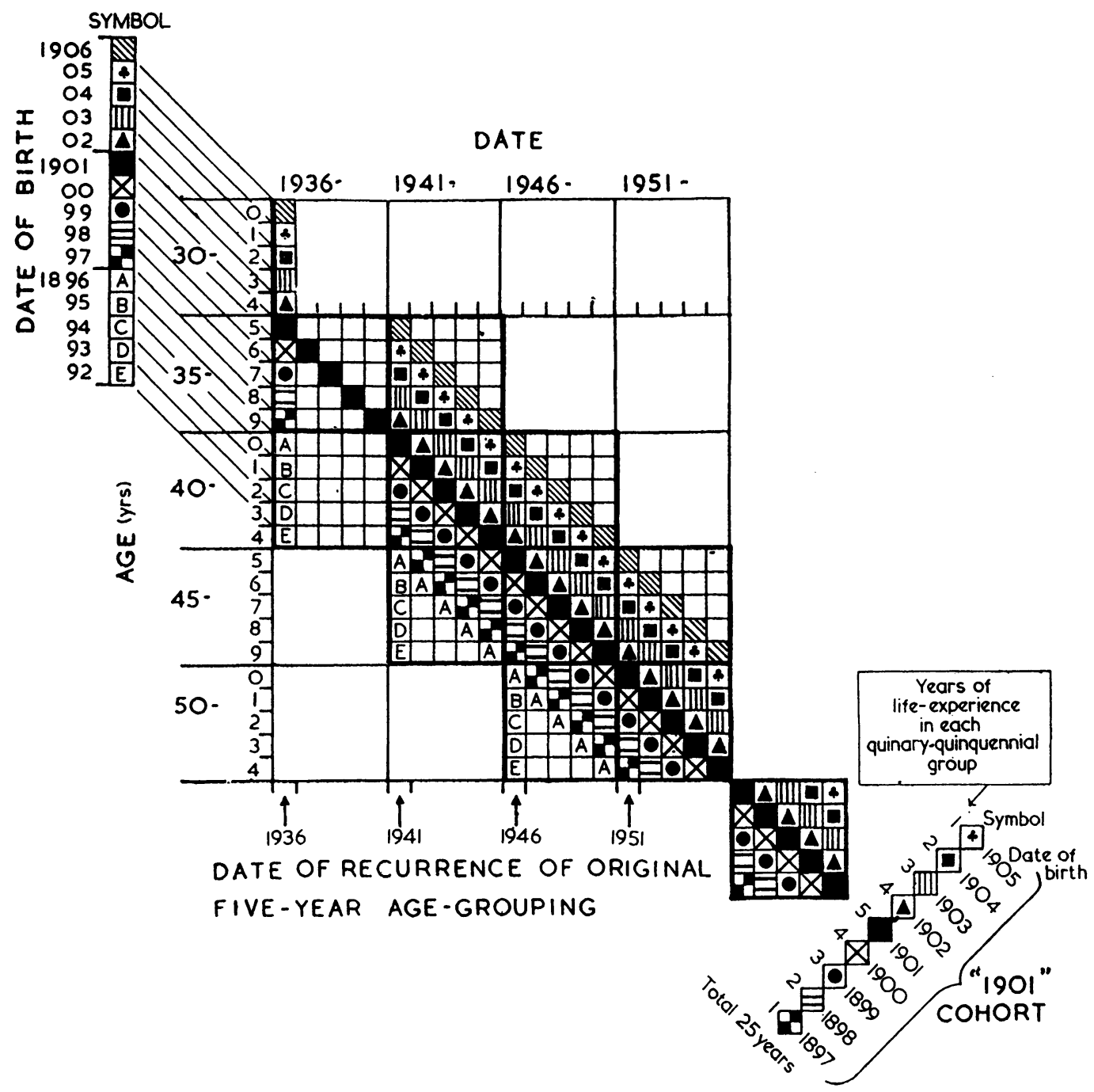

Figure 2 Cohort analysis by quinary-quinquennial grouping, showing how a cohort is formed. In this example the year 1901 is the central year of the cohort; it contributes more life-experience than any other year and does not appear in any other cohort.

membership of a group aged between 50 and 55 in 1951, even if no deaths have occurred.

This point is perhaps best shown by a graphical methods, as in Fig. 2.

If we allot a different birth symbol to each of the fifteen years 1892 to 1906 and then plot the movement of these symbols to the appropriate ages in a series of calendar years, we can see that birth-dates can be followed.

(a) by following one year at yearly intervals,

(b) by taking any group of $n$ consecutive birthyears and examining their experience every $n$ years, when that grouping will recur,

(c) by taking any group of $n$ consecutive birthyears and combining the experience in $n$ year groups. This will give a recurring pattern consisting of $n^{2}$ years of experience covering a range of $2 n-1$ years group around a central year which contributes $n$ years to the experience, and each year successively remote from this central year in each direction will contribute $n-1$, $n-2 \ldots \ldots[n-(n-1)]$, i.e. 1 . This formula is general and Method (a) is in fact a special case of it, in which $n$ is 1 .
From a practical point of view, Method (a) is not generally possible because of lack of data, and Method (b), used by Frost (1939) and Picken (1940), can be useful but is wasteful of data: a point which becomes important when studying mortality from individual causes of death. Method (c) is the one that has been most widely used, though none of the authors quoted has stated explicitly how the group experience was made up, and is the one which will be used in this paper: $n$ has been set at 5 (quinary-quinquennial grouping), since this is the smallest age group used by the RegistrarGeneral with sufficient consistency. The identifying birth-date of each cohort will be that of the central year, which will contribute 5 years of life experience to the quinary-quinquennial block and will not appear in any contiguous cohort.

When the mortality rates are arranged in this way, the grid shown in Fig. 1 may be considered as a surface, the third dimension being the agespecific death rate (Fig. 3).

This surface may be tranversed in any of the three ways already described for the grid, but only the diagonal path will describe the 


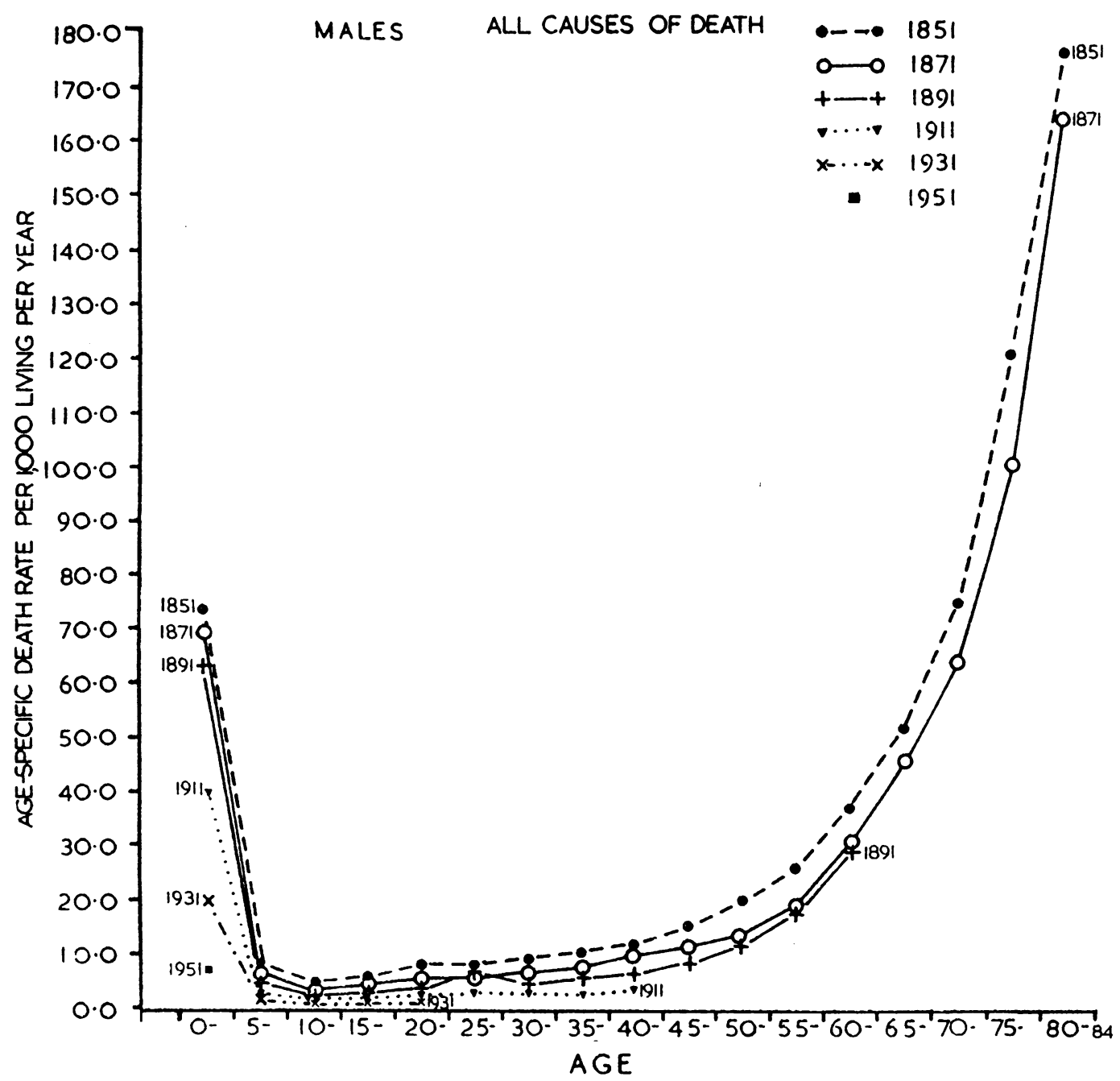

Figure 4A Age-specific death rate in England and Wales (All Causes) per 1000 living per year for the cohorts $1851,1871,1891,1911,1931$ and 1951. Males.

"shadow of death" that is threatening the survivors of any one cohort on their march from the cradle to the grave.

Errors and inaccuracies in the vital statistics will affect the mortality rates that are entered

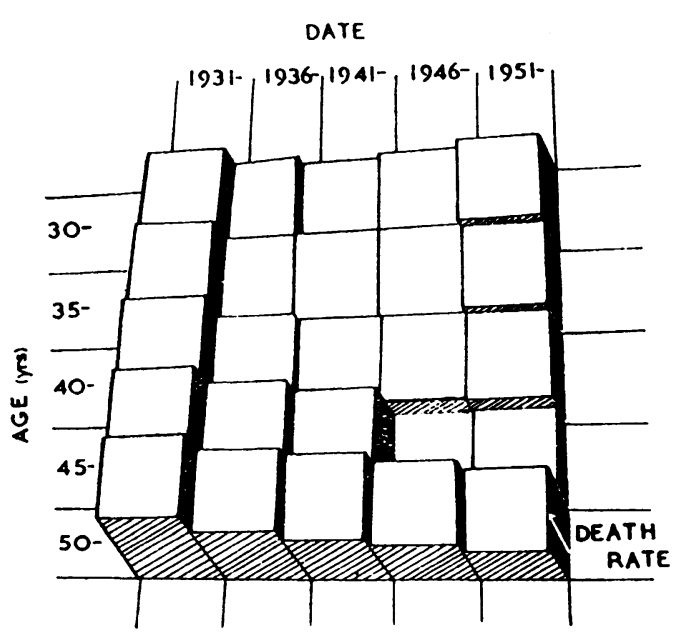

Figure 3 The age-specific death-rate grid considered as a surface. in each square. Since the same entries in the squares are used, although in a different sequence, whichever way the grid is transversed, none of the three ways of traverse is unaffected by such errors. The error that might specifically affect the cohort method is that which has been introduced by emigration and immigration. Although this effect cannot be measured or corrected, it is not believed to have been serious in England and Wales during the period covered by these studies. In some countries this difficulty might prove an insuperable barrier to the use of cohort analysis. If this were to be so, no other method of traversing the surface could be relied on to make a reasonable biological statement about changing mortality patterns in that country.

\section{The UNDERLYING CONCEPTS}

We are now in a position to return to the introductory paragraph and to elaborate the ideas contained therein. A considerable part of the literature quoted has described cohort analysis from the actuarial standpoint, making the assumptions that the mortality curve 


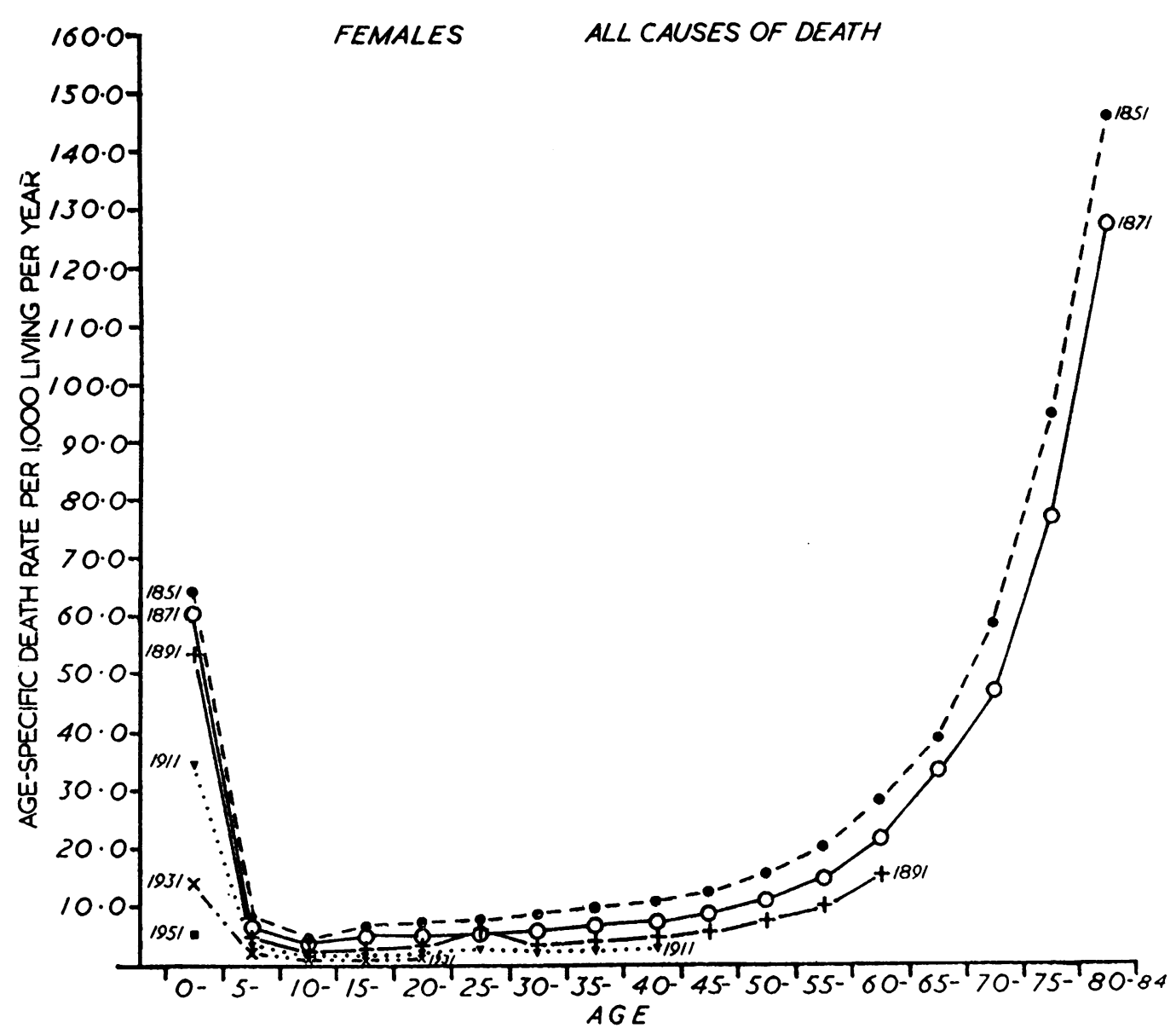

Figure 4B Age-specific death rate in England and Wales (All Causes) per 1000 living per year for the cohorts $1851,1871,1891,1911,1931$ and 1951 . Females.

changes smoothly with advancing age and that, if the curve can be graduated, it will be possible to extrapolate at least to a limited extent. The need for graduation and extrapolation has its roots in the demands of insurance companies for guidance in fixing insurance and annuity rates, and is not concerned with strictly biological considerations. In these circumstances it is but natural for actuarial writings are often irrelevant to questions that interest biologists.

Experience of the series of death rates traversed by the vertical lines (date-contours) had suggested that an equation of the general form $\mu \chi=\alpha+\beta c^{\chi}$ (Makeham, 1860) might adquately describe the shape of the curve traced by the force of mortality $(\mu \chi)$ at any age $\chi$. As often happens, a convenient shorthand description of shape was assumed by some to be a law of nature, and attempts were made to graduate or smooth the cohort mortality experience in order to find the terms of this equation and to extrapolate the curve from a "law of mortality". It may well be that there is in fact a hard core of mortality which improved environmental and treatment will leave untouched; indeed, unless immortality is invoked, there must be some such core. It may also well be that this residual mortality will obey some mathematical law. It is very doubtful whether the mortality rates that have already been recorded approach this hypothetical "hard core". If they do not, the cohort mortality curves as they stand will be a better record of the effects of social history than the graduated curves.

The other pre-occupation of some of the authors quoted was that, whilst the pattern of mortality, either general or disease-specific, did in fact follow a pre-ordained course, even if not that prescribed by the Makeham type of formula, the intensity of the attack on the community varied from time to time. This variation was assumed to be fixed by the intensity of attack during the early years of life. The definition of the word "early" in this context varied from author to author.

Despite Karl Pearson (e.g. 1912) and his school, there is no reason to doubt that a record of early good health reflected by low death rates at early ages may in general predispose to continued good health reflected by lowered death rates at higher ages. At the same time it must be remembered that environmental and therapeutic changes occur continually during the life-span of each cohort. It is extremely likely that this continuous alteration will result in mortality changes other than those that can be attributed to influences as work in the early (by any definition) years of life.

If, then, cohort mortality can be fairly considered as the product of at least three factors: 
$5 A$

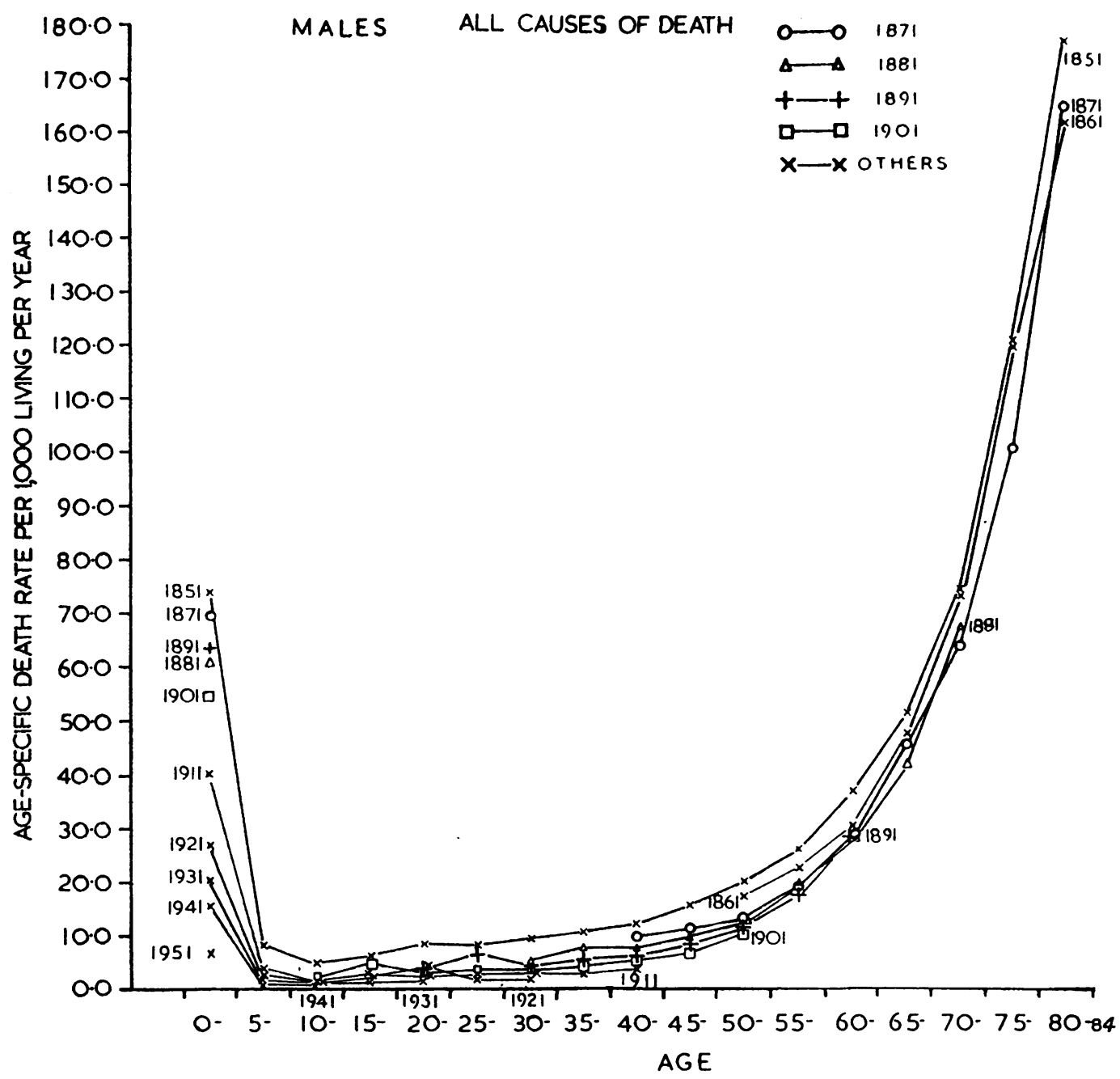

Figure 5A Age-specific death rate in England and Wales (All Causes) per 1000 living per year for the cohorts $1871,1881,1891$, 1901; with some additional detail from the cohorts 1851, 1911, 1921, 1931, 1941, and 1951. Males.

(1) that which is biological and inescapable,

(2) that which is due to early nurture,

(3) that which is due to constantly changing environment and therapy (i.e. later nurture),

it follows that much of the previous use of cohort analysis has neglected to exploit its full possibilities, and has been based on an incomplete statement of the nature-nurture complex.

A profitable, and largely unexploited, use of the method would be to say: "The cohort analysis of a series of death rates shows certain changes over the years. The social history of the community concerned shows certain changes over the years. The changes in social history have led to the setting up of certain hypotheses linking them with changes in death rates. Are the changes in death rates revealed by the cohort analysis compatible with these hypotheses or not?". This would not be a shortterm study and it would need a synthesis of the study of social conditions, the history of medicine and surgery, and of carefully complied Tables of age-specific disease-specific death rates in a form suitable for cohort analysis. Haenszel and Shimkin (1956) have already conducted a limited investigation on these lines.

Some knowledge of the time-lag between putative cause and effect will also be required. If the cohort analysis of mortality rates is regarded as depicting ripples on the pond of human experience, and if these ripples are to be attributed to a particular brick being thrown into the pond, we must make quite certain they were not in fact there before the brick reached the surface of the water.

A necessary corollary to accepting the cohort analysis of a series of death rates as being the best biological interpretation of those given by the three methods of traverse is that we must abjure the vertical series (date-contour) as a basis for the formation of biological theories, unless the rates at each age over the whole series (age-contours) are constant, in which case the date-contours and the cohort series become identical. If enough data have been collected to investigate whether or not the rates at each age are constant, it is probable that 


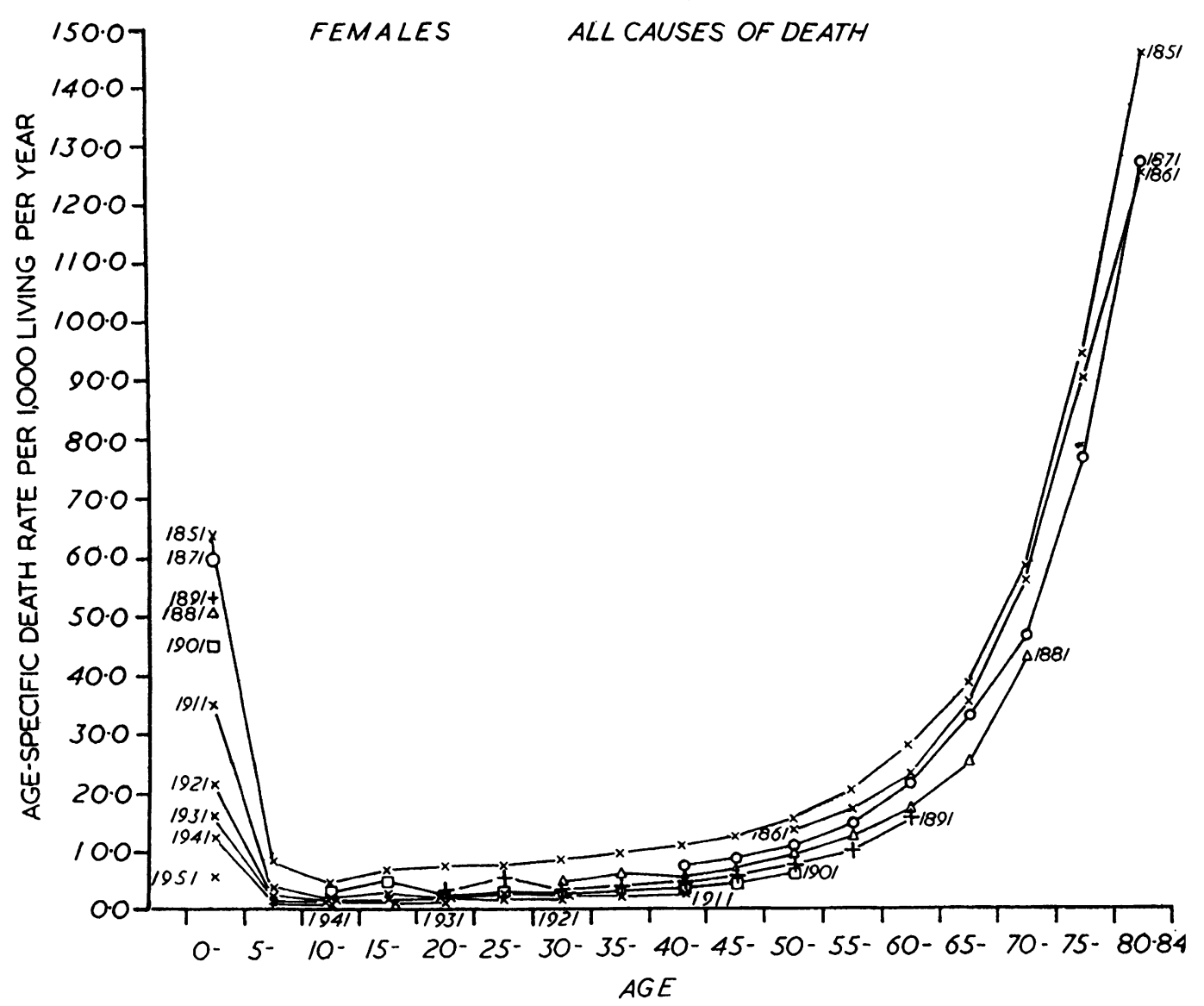

Figure 5B Age-specific death rate in England and Wales (All Causes) per 1000 living per year for the cohorts $1871,1881,1891,1901$; with some additional detail from the cohorts 1851, 1911, 1921, 1931, 1941, and 1951. Females.

enough data have been collected to make the cohort analysis. If they have not been collected, there is no logical justification for using the date-contour faute de mieux.

\section{EXAMPLES OF RESULTS}

\section{General Mortality Rates in ENGLAND aND WALES}

Figs $4 \mathrm{~A}$ and $4 \mathrm{~B}$ show the age-specific death rate per 1000 living per year for males and females.

Six cohorts, formed as previously described for quinary-quinquennial grouping, are used. The central years are spaced 20 years apart. The rates are calculated from populations and deaths that include non-civilians. The data are extracted from the successive volumes of what started as the "Annual Reports for the Years 1911-20" of the Registrar-General (1913-22) and continued as the "Statistical Reviews of England and Wales for 1921-54" (RegistrarGeneral 1923-55). Data relating to the years before 1911 were abstracted from the "Annual Report" for 1911.

In general, each successive cohort shows a lower death-rate than its predecessors at all ages for which the rates have been calculated. In detail, this is not entirely true, because cohort 1891 shows a kink in both male and female mortality at age 25-29. This kink, which is of sufficient size to cross the line of cohort 1871 at that age, is due to the influenza epidemic of 1918-19.

The lowering of the motality rates at later ages is clearly not entirely dependent on the lowering of mortality rates at ages under 5 , for there is very little improvement at these ages between the cohorts 1851, 1871, and 1891, whilst there is a marked improvement at, say, age 45 to 49 . Infantile mortality (i.e. that under 1 year, not shown in the Figures) did not begin to drop until after 1901, but improvements at higher ages were established for cohorts born before then. Thus, whilst it is possible to deny the argument of Pearson (1912) that a lowering of the infantile mortality rate must be inevitably followed by raised mortality rates in the juvenile range, it is not possible to invert this argument, and to say that improvements in mortality rates at any age must necessarily be preceded by improvements in the mortality rates at an earlier age in respect of any two cohorts being compared.

In Figs 5A and 5B, cohorts spaced less widely have been used, though not all are drawn over the whole extent of their courses lest graphical confusion result. The effect of the 1918-19 influenza epidemic can now be seen as a kink at age $15-19$ in the 1901 cohort, at $25-29$ in 


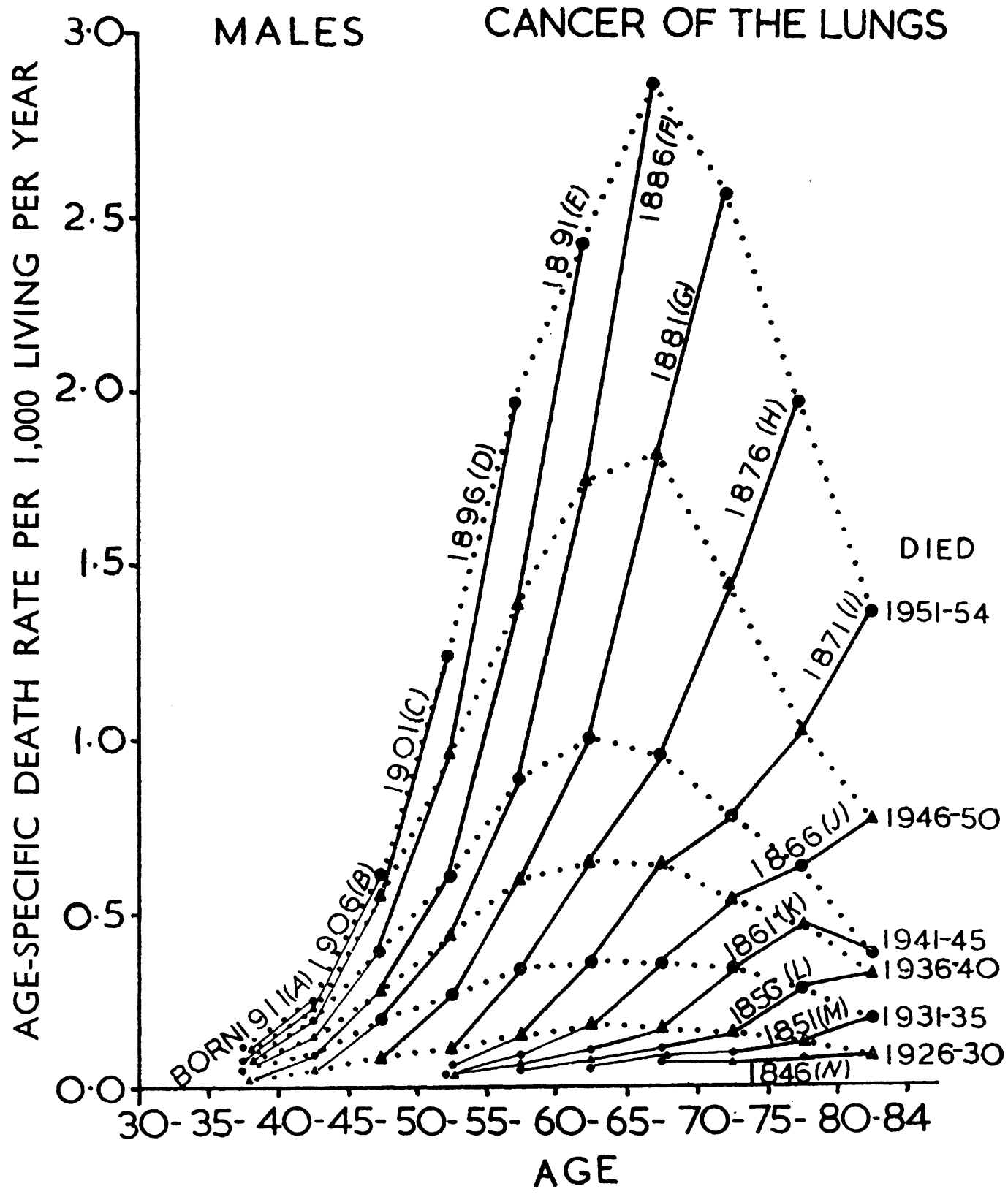

Figure 6A Cohort and date-contour analysis of changing rate. Age-specific death rate in England and Wales (Cancer of the Lungs) per 1000 living per year, in quinary-quinquennial groups from 1846 to 1911, arranged according to date of birth (cohort analysis) and date of death (date-contour analysis) for males. The rapid rise exceeds the critical level.

the 1891 cohort, and at $35-39$ in the 1881 cohort. It is still detachable, though masked by the rapidly rising force of mortality, at age $45-49$ in the 1871 cohort. The persistence of this kink in both the male and female cohorts differentiates it from an increased mortality affecting men who served in the 1914-18 war. The use of a quinquennial group underestimates the true severity of the epidemic. This detailed analysis also shows that several of the male cohort lines overlap at higher ages, or show but little improvement; this tendency is hardly to be seen amongst the female cohorts.

Thus, whilst a change in mortality has taken place during the last century, this change has not been entirely predetermined by the mortality of the earliest years of life, nor has it always been constant in direction. Although man is unique amongst animals in being able to generate intentionally a constantly changing environment, he is not prescient, and there is no reason to believe that his changes will always be beneficial. There is therefore no compelling reason arising from these studies to believe that the occasional deterioration in the male death rates at higher ages is a reflection of the late action of deleterious genes or is otherwise determined by failure of selection at earlier ages, since an adverse adult environment of relatively recent origin could also produce this change. There is also no compelling reason to reject the former hypothesis; elucidation must be sought elsewhere.

In most of what has gone before, the discussion has been confined to general mortality. When individual causes of death are con- 


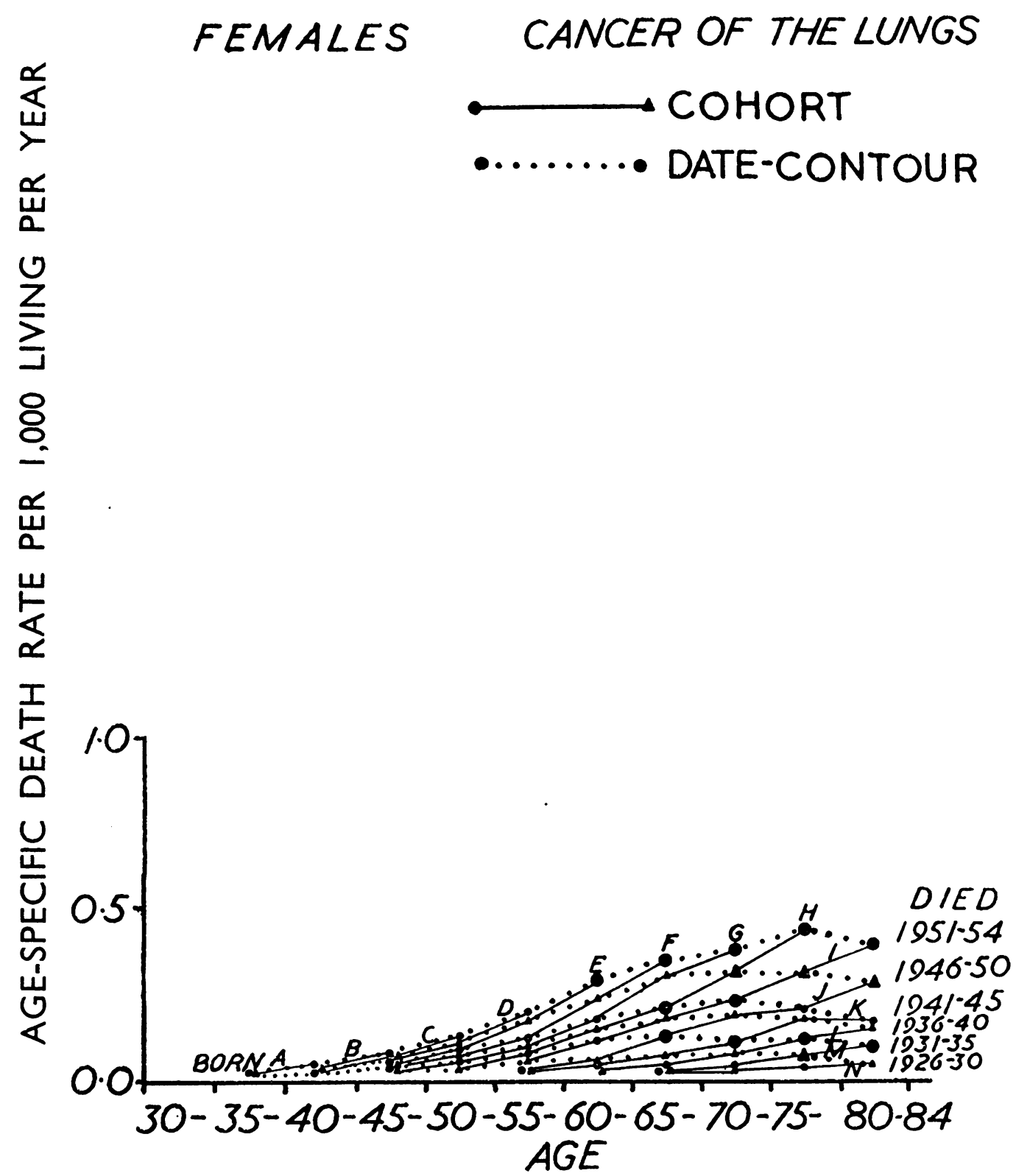

Figure 6B Cohort and date-contour analysis of changing rate. Age-specific death rate in England and Wales (Cancer of the Lungs) per 1000 living per year, in quinary-quinquennial groups from 1846 to 1911 , arranged according to date of birth (cohort analysis) and date of death (date-contour analysis) for females. The slower rise just attains the critical level.

sidered, there is even less reason to believe that for many diseases the "hard core" of the mortality pattern has been reached. How far early environment will be effective in fixing the mortality pattern, and how far later environment and therapy will affect this pattern will vary considerably from disease to disease. The picture revealed by cohort analysis for different diseases must be assessed in the light of what is known about each one.

Effect of a Changing Rate on the Mortality PATTERN AS SHOWN BY COHORT ANALYSIS and Date-Contour ANAlysis

The interpretation of mortality patterns revealed by date-contour analysis can be made more simple, and certain pitfalls can be avoided, if the effects of a changing rate are realized. The examples shown here are taken from the cancer field, not only because one of them has already been discussed by Korteweg (1951, 1953), but also because the relatively simple form of the mortality pattern for cancer, and the relatively slow fluctuations that are exhibited, make these illustrations almost as simple as one could obtain by using hypothetical data.

(1) Effect of a Rising Rate - Figs 6A and 6B show all the squares of the quinary-quinquennial grid plotted and joined along the cohort line and along the date-contour line for the recorded mortality for cancer of the lung in England and Wales from 1911 to 1954.

In males the date-contour line shows the highest death rates to lie between the ages of 


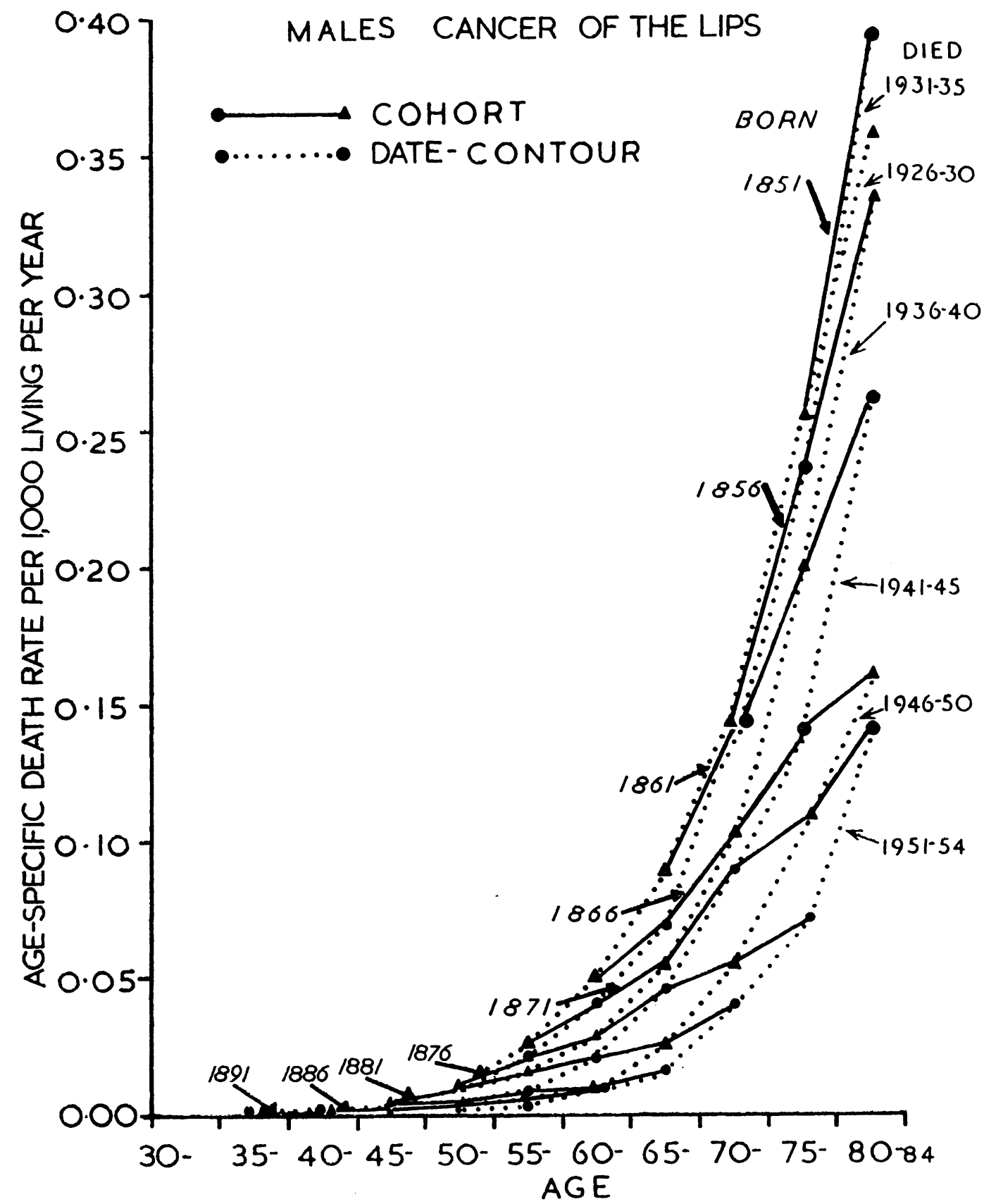

Figure 7 Cohort and date-contour analysis of changing rate. Age-specific death rate in England and Wales (Cancer of the Lips) per 1000 living per year, in quinary-quinquennial groups from 1851 to 1891 , arranged according to date of birth (cohort analysis) and date of death (date-contour analysis) for males. The rapid fall produces a marked inflexion in the date-contour line.

60 and 70 , but the cohort line shows a continuous rise. The effect will always be produced when the rise from date-contour to date-contour exceeds a critical ratio to the rise from age group to age group in each cohort. This is the effect described by Korteweg $(1951,1953)$.

In females the date-contour line flattens out and shows an apparent lock of age specificity, but the cohort line reveals a ready rise. In this example the rise from contour to contour is only just attaining the critical level required to produce an apparent modality in the datecontour line.

(2) Effect of a Falling Rate - Fig. 7, illustrating the mortality from cancer of the lip for males in England and Wales, shows the effect of a falling rate. The date-contour line shows a hollowing out, giving an inflection in the middle of the range which is not found in the cohort lines.

If we wish to consider the effect of a rising or falling rate on more complex curves, such as the modal type of curve shown by tuberculosis mortality (Springett, 1950), the same principles of curve analysis can be applied to each segment.

These two illustrations show how important it is not to base biological theories on the shape of the mortality curve derived from the datecontour unless it is known that the rates have been substantially unchanged during the time covered by the age range under consideration.

An example of such a theory, recently advanced, is that of Nordling (1953), who uses the date-contour of the date rates from cancer of all sites to suggest that, since this yields a 
log-logarithmic plot reasonably described by the exponential of the sixth power, cancer might be the result of a seventh mutation. Stocks $(1953 \mathrm{a}, \mathrm{b})$ recognizes that such a theory should be based of cohort analysis, but does not explore the cancer field sufficiently widely to demonstrate that, if such a theory could be based on death rates, it must apply to only a few sites of cancer, or that the theory must be so qualified that the postulated number of mutations would vary not only from site to site, but also for the same site at different times.

It is not the author's intention to deny that any hypothesis invoking a sequence of changes or mutations as a necessary precursor to malignant change could be valid, but only to point out that premises based in this way on the datecontour analysis of mortality rates offer at the best a very insecure foundation for advancing such a hypothesis.

\section{SUMMARY}

A history of cohort analysis has been given, and it has been pointed out that the bulk of the literature on the subject has dealt with concepts that assume either that graduation and possibly extrapolation are desirable, preferably in conformity with some formula expressing what is implicitly accepted as a "law of mortality", or that, whether or not a fixed pattern of mortality exists, the intensity of mortality risk is largely determined in early life.

The view is advanced that either concept alone, is, or both concepts together are, inadequate and may lead to an improper assessment of the nature-nurture complex, since environment and therapeutic measures are constantly changing. The plea is made for the technique of cohort analysis to be used as a narrative or historical technique, and for a synthesis of knowledge derived from social history, medical history, and cohort analysis to be made to interpret the narrative.
The effects of a changing rate on the mortality pattern as depicted by date-contour analysis and cohort analysis are outlined and the danger of basing biological theories on the mortality pattern revealed by date-contour analysis alone is indicated. This point is illustrated by reference to a recent theory that has been based on such an analysis.

This investigation received support from grants to the Chester Beatty Research Institute from the British Empire Cancer Campaign, the Jane Coffin Childs memorial Fund, the Anna Fuller paign, the Jane Coffin Childs memorial Fund, the Anna Fuller Service.

\section{REFERENCES}

Andvord KF. Norsk. Mag. Laegevid 1930;91:642.

Barclay RS and Kermack WO Proc. roy. Soc. Edinb. 1937-38; 58:55.

Cramér H and Wold H. Nordic Statistical f 1934:5:3.

Cramér H and Wold H. Skand. Aktuar Tidskr 1935;18:161.

Clemmesen J, Nielsen A, and Jensen E. Acta Un. int Cancr 1953;9:603.

Cutler SJ and Loveland DB. F. nat. Cancer inst. 1954;15;201.

Daw RH. F. Inst. Actu. 1950;76:143.

Dorn JF. Acta Un. int, Cancer 1953;9:552.

Derrick VPA f Inst Actu, 1927:58:117.

Editor's Footnote. Acta Un. int. Cancr. 1953;9:528.

Frost WH. Amer. F. Hyg. 1939;30:Sect. A. 91.

Frost WH. Amer. F. Hyg. 1939;30:Sect. A. 91.

Greenwood M. F. roy. statist. Soc. 1936;99.6

Gompertz B. Philos Trans. 1825;115:513. 1417.

Kermack WO, McKendrick AG and McKinlay PL. Lancet 1943a;1:698

Kermack WO, McKendrick AG and McKinlay PL. F. Hyg. (Camb) 1943b;34:433.

Korteweg R. Z. Tuberk., 1927;49:176.

Korteweg R. Brit. F. Cancer, 1951;5:21.

Korteweg R. Acta Un. int. Cancr., 1953;9:529.

Lancaster HO Med. $f$. Aust. 1950;1:655.

Levin ML. Acta Un. int. Cancr. 1953;9:531.

Makeham WM. Assurance Mag. (Lond.). 1860;8:301.

Moine $M$ and Oudet P. Bull. Inst. nat. Hyg. 1947;2:241.

Moine M and Oudet P. Bull. Inst. nat. Hyg.
Pearson K. Proc. roy. Soc. B., 1912;85:469.

Pearson K. Proc. roy. Soc. B., 1912;85:469.

Picken RMF. Publ. Hlth (Lond.), 1940;53:145.
Registrar-General (1913 to 1922). "Annual Reports 74 to 83 for the Years 1911-20". HMSO, London.
fortar-General (1913 to 1922). "Annual Repo

for the Years 1911-20". HMSO, London.
Registrar-General (1923 to 1955) "Statistical Reviews of England and Wales for 1921-1954". HMSO, London.

Spicer CC. F. Hyg. (Camb.). 1954;52:361.

Springett VH. Ibid. 1950;48:361.

Springett VH. Lancet. 1952;1:575.

Stocks P. Brit f. Cancer. 1953a;7:283.

Stocks P. Ibid. 1953b;7:407.

Taylor W. British fournal of Preventative and Social Medicine. 1952;6:226. 